\title{
Repensar la economía y la sociedad postpandemia: Mujeres y vulnerabilidad
}

\section{Rethinking the post-pandemic economy and society: Women and vulnerability}

\author{
Bernardita Escobar* \\ ASOCIACIÓN CHILENA DE HISTORIA ECONÓMICA
}

\section{RESUMEN}

Este ensayo reflexiona específicamente la realidad de la pandemia y la profundización en la vulnerabilidad de las mujeres. Las mujeres representan la mitad de la población del mundo y tal como vamos a discutir, no existe tanta diferencia entre las mujeres que están en Chile, México y Latinoamérica, o en Europa, Estados Unidos y en Asia, es decir, el nivel de vulnerabilidad puede cambiar en algunos países, puede ser mayor que en otros, pero la situación global de afectación hacia la mitad de la población merece la importancia de ser tratado en primer orden.

Palabras clave: Pandemia, mujeres, vulnerabilidad, cuidados, política pública

\begin{abstract}
This essay specifically reflects the reality of the pandemic and the deepening vulnerability of women. Women represent half of the world's population and as we will discuss, there is not so much difference between women who are in Chile, Mexico and Latin America, or in Europe, the United States and Asia, that is, the level of vulnerability may change in some countries, may be greater than in others, but the overall situation of affectation towards half the population deserves the importance of being treated in the first order. Keywords: Pandemic, women, vulnerability, care, public policy
\end{abstract}

* beni.escobar.andrae@gmail.com 


\section{INTRODUCCIÓN}

reo que la pandemia ha hecho evidente cuáles son y en qué niveles de vulnerabilidad se encuentran determinados sectores de la sociedad que han estado vulnerables previamente, pero que la pandemia ha ahondado de manera muy cruel sus niveles de vulnerabilidad. Estos grupos son -al menos- las mujeres, los ancianos y en general la gente de menores ingresos y que viven situaciones de menores ingresos, grupos que serán doblemente afectados. La situación de los migrantes también, por lo menos en Chile, ha quedado sumamente manifiesta la vulnerabilidad, los niveles de pobreza y de abandono que muchas veces viven. También una dimensión distinta es la infancia y el efecto que va a tener la pandemia para los niños de distintas edades y en distintos niveles socioeconómicos, al tener que enfrentar sus tiempos de educación en reclusión. Eso yo creo que va a ser un tema importante que vamos a tener que aprender a mirar en su propio mérito y con los niveles de profundidad que esto amerita por la importancia del sector de población infantil, pero además con los aspectos de largo plazo que tiene, porque los otros sectores son adultos y las afectaciones son más bien temporales y no necesariamente de largo plazo.

\section{Vulnerabilidad de las MUjeres}

Quiero tratar específicamente los problemas que genera la pandemia a la vulnerabilidad de las mujeres. No es un tema menor porque las mujeres representan la mitad de la población del mundo y tal como vamos a discutir que no existe tanta diferencia entre las mujeres que están en Chile, en México y Latinoamérica, en Europa, en Estados Unidos y en Asia. Es decir, el nivel de vulnerabilidad puede cambiar en algunos países, puede ser mayor que en otros, pero la situación global de afectación hacia la mitad de la población merece la importancia de ser tratado en primer orden.

Es evidente que, en casi todas partes del mundo, Chile no es la excepción, las mujeres abarcan una producción altísima de la fuerza de trabajo que está en la primera línea, enfrentando la pandemia en el sector salud como enfermeras, como médicas, como profesionales 
auxiliares de la salud. Dentro de las funciones que se ejercen en los recintos hospitalarios y en general en el área de salud, pero en particular en el recinto hospitalario público y privado, las mujeres tienen una alta magnitud e importancia en la fuerza laboral y, por lo tanto, corresponde a un sector fuertemente compuesto por mujeres que están enfrentando evidentemente un alto riesgo de contagio.

Al margen del sector salud, las mujeres siempre han sido y en todas partes del mundo han recibido remuneraciones o compensaciones pecuniarias menores por las labores equivalentes que ejercen sus pares masculinos. Este solo hecho por sí solo convierte también a las mujeres en un sector mucho más vulnerable a ser despedido o desvinculado de sus labores porque son en general "más baratas" de ser despedidas. Las cifras de empleo que han salido, por lo menos en Chile, la encuesta de empleo de PyMes mostraba altos niveles de desempleo en general, pero concentrado de manera más fuerte en mujeres y también una fuerte caída en la participación laboral de las mujeres. Esto ocurre no solamente porque las mujeres reciben menos remuneraciones, sino que además están enfocadas también a sectores o funciones que están mayoritariamente ocupadas por mujeres que como tal reciben menores compensaciones. Además, existe un tercer efecto: debido a las diferencias de género en las funciones realizadas al interior del hogar que son realizadas mayoritariamente por mujeres. Todas las encuestas que yo he visto de uso de tiempo muestran que la cantidad de horas totales destinadas a actividades labores remuneradas y no remuneradas son mucho más altas en el caso de las mujeres. Esto conlleva reconocer que las labores no remuneradas que realizan adentro del hogar las mujeres les ha significado una responsabilidad social mayor de hacerse cargo de esas funciones y por lo tanto, en una situación de riesgo de salud vital de su familia o de sus seres queridos, las mujeres se retiran del mercado laboral para ejercer las labores de cuidado dentro del hogar con la familia directa, pero también por la familia extendida sin recibir ninguna compensación económica por hacerlo, ni tampoco previsional. Hay una falta de reconocimiento de ese esfuerzo y, por lo tanto, la situación estrictamente laboral es de alto cuidado. Las 
mujeres se retiran de la fuerza de trabajo y reciben menores ingresos por lo mismo, son mucho más proclives a ser despedidas en una situación de riesgo económico más bien sistémico.

Un hecho también en Chile recurrente es la exposición a la violencia de género y en particular a la violencia intrafamiliar que siempre ha sido un tema que se ha conocido, pero que ha sido un poco tabú y las cifras recién han permitido distinguir entre los crímenes que pueden ser catalogados como violencia intrafamiliar gracias a los indicadores de la Fiscalía Nacional que persigue los delitos en general, pero que ahora está obligada a catalogar la violencia intrafamiliar en una categoría específica, lo que ha mostrado que las mujeres son las principales víctimas de violencia intrafamiliar. La pandemia y el confinamiento ha expuesto a un riesgo mucho mayor de ejercicio de esta violencia hacia las mujeres en el formato de violencia interfamiliar. Las ha relegado a una situación de aislamiento tal que ni siquiera pueden pedir ayuda, o se encuentran con más dificultades de pedirla a las que se han enfrentado históricamente. Lo que aumenta la gravedad de esta situación, de por sí súper delicada, es que el Estado-nación no ha estado a la altura nunca y por eso esta situación incrementa la vulnerabilidad de las mujeres. Esto ha llevado o ha despertado incluso iniciativas de protección o de intento de protección desde el sector privado como esta iniciativa que se llama "mascarilla 19" y otras similares que me ha tocado ver, lo que deja en evidencia la necesidad de hacerse cargo de este problema, pero también expone el retraso o nivel de inacción o incapacidad del estado de proteger a las mujeres, o falta de voluntad por proteger a las mujeres. Pero lo más reciente que también muestra esta preocupación o falta de empatía o de preocupación multidimensional de parte de la autoridad de hacerse cargo de la violencia de las mujeres es el caso del grupo de mujeres "Las Tesis", que relevó una manifestación de protesta contra la violencia de género hace unos meses atrás que tuvo revuelo mundial y que hoy enfrentan una querella por parte de Carabineros de Chile por amenaza a estas mujeres. Esto muestra que el Estado, o mejor dicho, órganos del Estado, no tienen problemas en reaccionar 
para reprimir o coartar las manifestaciones de repudio que emergen desde las mujeres por esa violencia, criminalizándola. Me preocupa, porque en realidad cuando uno empieza a hacerse cargo de las señales sociales de descuido por la situación de vulnerabilidad de las mujeres uno no puede pasar por alto todos estos episodios que van configurando un cuadro que yo creo que es bastante preocupante.

Mujeres EN LA ACADEMIA, TRABAJO REMUNERADO Y TRABAJO DOMÉSTICO.

Las mujeres en el mundo de la academia tienen también su correlato exactamente con los mismos factores que yo acabo de mencionar y que ya se está constatando. Hace un par de semanas atrás leí al menos dos artículos académicos en el cual se evidencian los mismos factores que mencioné anteriormente. Las académicas tienen que hacerse cargo de las labores de cuidado al interior de su familia y de su familia extendida porque la sociedad les obliga digamos socialmente a hacerse cargo, pero sus parejas no se ven necesariamente afectados por esta carga. Pero tampoco sus pares académicos, porque los académicos hombres no enfrentan esta misma relación o contracción en la productividad académica que sí enfrentan sus colegas mujeres. Yo creo que esto es serio en sí mismo porque resulta que si no nos hacemos cargo de estos efectos, la progresión en la carrera académica de las mujeres también se verá afectada y si nosotros consideramos además que la calificación de los estudiantes es mucho más severas con las profesoras mujeres que con los profesores, como señalan varios estudios, las evaluaciones van probablemente a ahondar los rastros de diferencia de género que ya traíamos y que no son solamente culpa de la pandemia. Estos signos nos revelan que no nos debiera sorprender ver que las profesoras van a enfrentar evaluaciones más severas de parte de sus estudiantes producto del malestar que van a tener los estudiantes por haber enfrentado un semestre en una situación compleja, pero que tienen que rendir y demostrar productividad como si no lo tuvieran. La pandemia entonces representa una amenaza real y tangible que es multidimensional respecto de la situación de vulnerabilidad de las mujeres. 
Ahora, si pensamos en una situación más retrospectiva, hay que preguntarse cuáles son las políticas que podemos echar a mano para hacerse cargo de políticas públicas en situaciones de pandemia. Lo que es preocupante es que nuestros países enfrentaron pandemias antes y sin embargo jamás he visto un programa de clases que incluyera las pandemias de la viruela en el siglo XVIII ni otras crisis sanitarias como las del siglo XIX. Tuvimos varios episodios serios, pero la pandemia de 1918 sencillamente no estuvo en el currículum de clases, tampoco en la universidad, nada sobre las políticas económicas que se enfrentaron a partir de la pandemia y lo que ocurre en el caso particular de Chile respecto a la pandemia de 1918 es que cualquier política económica que se pudo tomar para enfrentar el desastre que significó de la muerte cerca del 1\% de la población de aquella época, fue que coincidieron con las políticas de reforma del Estado que se desarrollaron producto de lo que en Chile se llamó la cuestión social que fue un llamamiento que hizo el doctor Augusto Orrego en 1880 en una serie de cartas donde manifestaba la precariedad que vivían sectores de la sociedad que estaba completamente al margen de la política pública. Eso llevó, aunque lentamente, a ir haciendo una reforma del Estado hacia mayor protección de las personas, y ellas coinciden temporalmente con las reformas que puedan haber derivado de la pandemia de 1918. La pregunta que surge, es cuánto aprendió la sociedad chilena de esa pandemia para hacerse cargo de los problemas de la cuestión social; ellas se confunden y no son fáciles de identificar entre sí. En definitiva, la pandemia de 1918 y sus repercusiones económicas no ha formado parte, aunque podría ser interesante, de los currículums de la historia económica de Chile.

Yo creo que el efecto de la pandemia sobre las mujeres va a tener algo parecido, es decir, las mujeres traíamos una situación de vulnerabilidad expuesta y evidente a los niveles tan altos de estar expuestas a ser muertas por su pareja o por sus padres o por sus hermanos, y sin embargo, hemos llegado al año 2020 con esa vulnerabilidad abierta. Por lo tanto, no creo que vaya a ser tan fácil poder distinguir o identificar de manera más evidente cualquier cambio que pudiese producirse en reducción de la vulnerabilidad 
de las mujeres como resultado de enfrentar la pandemia. En general, soy bastante pesimista respecto a la capacidad de esta pandemia de remecer los fundamentos en los cuales está levantada esta sociedad porque la vulnerabilidad de este sector ante la población que son las mujeres va a competir con otras vulnerabilidades, como las de los sectores más empobrecidos y los sectores que tienen políticas públicas sobre las cuales fundarse para sobrellevar la pandemia. La vulnerabilidad de esos sectores amenaza de alguna manera la escasa estabilidad política y económica que existe dentro de nuestros países y en particular en Chile. Pienso que la autoridad va a tender a privilegiar las políticas de corte universal como lo que estamos viendo en Chile, las cuales soportan niveles de ingresos básicos a altos sectores de la sociedad y, por lo tanto, tienen un alto impacto fiscal y el equilibrio macroeconómico. De este modo, va a estar puesto sobre la balanza respecto a la capacidad de dar abasto o dar cobertura de protección mínima a estos amplios sectores de la sociedad. No me extrañaría que nuevamente la vulnerabilidad de las mujeres sea puesta debajo de la alfombra. Una señal de este elemento es lo que se ha visto en la discusión respecto de extender el posnatal a las mujeres y sustituirlo por el seguro de protección al desempleo que se está extendiendo para que hombres y mujeres que tienen hijos menores de siete años puedan tomar esta alternativa con cargo a un seguro financiado con el trabajo de las mismas personas y no por los fondos generales de la nación, y excluyendo entonces a sectores de mujeres trabajadoras que no tienen acceso a ese seguro dejándolas nuevamente desvalidas y enfrentadas a tener que depender de sus parejas que puedan tomar el seguro de cesantía, si es que tienen acceso, para enfrentar los efectos económicos de la pandemia.

\section{Conclusión}

Soy pesimista respecto de la capacidad y voluntad política de la sociedad para enfrentar las vulnerabilidades preexistentes de amplios sectores de la sociedad. ¿Qué podemos esperar entonces de la postpandemia? Yo creo que se puede generar división mayor entre la sociedad por muestras de falta de empatía por los menos afectados por la pandemia, y por una exacerbación de su vulnerabilidad 
por parte de los más afectados. El hecho de pretender funcionar durante la pandemia como si ella no existiera, en cierta medida, por ejemplo, con las clases online o sosteniendo el teletrabajo ha tensionado enormemente la vida cotidiana de todas esas personas porque es absolutamente imposible realizar las mismas labores en la misma cantidad de tiempo cuando además hay que hacerse cargo del cuidado de los hijos, de cocinar y todo lo que demanda vivir estando confinado. Por lo tanto, pretender cumplir con las mismas 44 horas de docencia y de investigación cuando las horas disponibles del día siguen siendo las mismas yo creo que tensiona a todas las personas que han podido mantener su trabajo y han podido mantenerse trabajando con teletrabajo. Pretender que esto no existe es lo que está detrás de lo que está ocurriendo y creo que está generando tensión en la sociedad.

Para finalizar, creo que la intromisión de la esfera del trabajo en el de la intimidad del hogar, producto del teletrabajo significa un mayor riesgo también de fractura social entre los sectores que tienen una posibilidad de aislar la esfera laboral de la esfera de la intimidad de sus hogares de quienes no lo pueden hacer. Esto está mediado por las condiciones de vida y de construcción material del hogar de las personas. Afecta negativamente principalmente a las personas que tienen menos metros cuadrados en el hogar, familias con menores de edad o personas enfermas que requieren mayores cuidados y que están por encima del resto en niveles de fragilidad. Esto va a mostrar una fractura entre los sectores que están más afectados directamente, que están más imposibilitados de hacer como si no hubiera ocurrido la pandemia respecto de los que sí lo pueden hacer. El hecho de tratar de sobrellevar rápidamente los problemas del confinamiento y no hacerse cargo de las fuertes infracciones a la cotidianidad e intimidad que implica el teletrabajo, incluso en aquellas personas que no están siendo afectadas de manera directa, muestra el alto nivel de tensión y de fractura social que puede generar la pandemia en el futuro cercano, incluso entre los afortunados, que no se enfermaron, y no perdieron el empleo. 
Fecha recepción: 2 de julio de 2020

Fecha aceptación: 20 de julio de 2020

Versión final: 20 de octubre de 2020 